\title{
Make like a glass frog: In support of increased transparency in herpetology
}

\author{
Benjamin Michael Marshall ${ }^{1} \&$ Colin Thomas Strine ${ }^{1}$
}

${ }^{1}$ School of Biology, Institute of Science, Suranaree University of Technology, Nakhon Ratchasima, Thailand

\begin{abstract}
Across many scientific disciplines, direct replication efforts and meta-analyses have fuelled concerns on the replicability of findings. Ecology and evolution are similarly affected. Investigations into the causes of this lack of replicability have implicated a suite of research practices linked to incentives in the current publishing system. Other fields have taken great strides to counter incentives that can reward obfuscation -chiefly by championing transparency. But how prominent are protransparency (open science) policies in herpetology journals? We use the recently developed Transparency and Openness Promotion (TOP) Factor to assess the transparency promotion of 19 herpetology journals, and compare the TOP scores to broader science. We find promotion of transparent practices currently lacking in many herpetological journals; and encourage authors, students, editors, and publishers to redouble efforts to bring open science practices to herpetology by changing journal policy, peer-review, and personal practice. We promote an array of options -developed and tested in other fields- demonstrated to counter publication bias, boost research uptake, and enable more transparent science, to enrich herpetological research.
\end{abstract}

Keywords: accountability, herpetology, journal policy, open science, peer review, reproducibility, transparency

\section{INTRODUCTION}

$A^{c}$ cross scientific disciplines replication efforts have revealed marked deviation from previously observed results (Freedman et al., 2015; Kelly, 2019; Open Science Collaboration, 2015); this lack of replicability, as shown in medical fields, can incur huge costs and impact human health (Freedman et al., 2015). Despite relatively infrequent efforts to test replicability in ecology and evolution (Kelly, 2019; Schnitzer \& Carson, 2016), several examples exist of apparently well-documented effects being brought into question by larger scale replications and meta-analyses (Clark et al., 2020; Roche et al., 2020; Sánchez-Tójar et al., 2018; Wang et al., 2018) suggesting that ecology and evolution must be similarly wary of irreplicable results.

The research community has begun questioning the causes behind observed inconsistencies in results, and exploring options on how to guarantee a more verifiable body of findings. Building a robust body of literature avoids wasting limited research resources (Grainger et al., 2019), correctly informs decisions, and maintains wider trust in science (Anvari \& Lakens, 2018).

Partly as a result of fears over irreplicability, we are seeing a change in how researchers and publishers work, namely a shift towards greater transparency and openness in published results. Shifts to greater transparency can counter a suite of incentivised questionable research practices -ranging from the seemingly benign, through questionable, to the demonstrably unethical (Ware \& Munafò, 2015)- that are suggested to amplify the variation in, and undermine the replicability of, results. The publishing system incentivises researchers to produce novel, significant results that can be presented within a clean singlearticle narrative (Brembs, 2019; Fanelli, 2012; O'Boyle et al., 2014). In particular we see prestigious journals prioritising exciting, novel studies with large effects, rather than those undertaking rigorous and replicable science (Barto \& Rillig, 2012; Brembs, 2019).

Despite near universal desire to follow best practice in research, the system's prioritisation of significant results (inadvertently) encourages detrimental research practices such as p-hacking, HARKing, and cherrypicking (Cairo et al., 2020; Forstmeier et al., 2017; Fraser et al., 2018). P-hacking is repeatedly testing the same data using different methods until a "significant" result is obtained, paired with failure to report repeated testing. HARKing (Hypothesising After Results Known) is reporting an unexpected result as expected, a way of ensuring a confirmatory result, possibly rationalised by "hindsight bias" on the part of the researchers (Forstmeier et al., 2017). Cherry-picking covers scenarios where researchers fail to report all variables or data 
tested, consigning non-significant variables or outliers to a file-drawer. All three practices are implicated in causing irreplicable results in other fields. Ecology and evolution, and by extension herpetology, are not insulated from the incentives encouraging detrimental research practices (Fraser et al., 2018).

Open science (transparency) provides a way of countering these questionable practices, while also presenting an opportunity to increase the value of scientific articles. Scientific articles often serve as currency for research careers (Rice et al., 2020), and play a central role in incentivising research practices good and bad (Brembs, 2018; Fanelli, 2010; Nosek et al., 2012). Implementing transparent practices in articles benefits researchers by building a more efficient workflow, boosting citation rates, increasing publication chances, and enhancing research reputation (Allen \& Mehler, 2018; Markowetz, 2015; Piwowar \& Vision, 2013).

Integrating more transparent practices into research is feasible without journal support, but journal policy can enhance normalising transparency (Nilsen et al., 2020; Roche et al., 2015). Aligning journal policy to normative ends may not be new, but recent efforts to combat opaque practices are exemplified by the Transparency and Openness Promotion (TOP) guidelines (Nosek et al., 2015); http:/(cos.io/top). The guidelines aim to help journals implement transparent policies, while also providing a framework (TOP Factor) for assessing journals' adherence to transparent policies. Although moves towards more transparent practices are new, the implemented solutions do indeed appear to be reducing publication bias and associated false positive rates (Allen \& Mehler, 2018; Kaplan \& Irvin, 2015; Scheel et al., 2020; Toth et al., 2020).

Herpetology - the study of reptiles and amphibianscovers a broad spectrum of disciplines, from anatomy and physiology, to genetics, evolution, and ecology. If there are replicability issues in the broader fields, then they likely exist in herpetology, yet journal practices targeted at mitigating reproducibility issues have yet to be examined. To understand to what extent herpetology journals currently promote open science practices, we explored how current journal policies adhere to the TOP Factor framework. We then use these findings to promote a number of changes, demonstrated by other fields, to improve openness and hopefully enrich herpetology.

\section{METHODS}

We produced a list of herpetology journals by searching for herpeto* using Scimago Journal and Country Rank (https://www.scimagojr.com/ accessed 2020-02-21). We attempted to retrieve author guidelines from each journal's website. For those journals with accessible author guidelines, we scored them according to the TOP Factor (final $n=19 ; 3$ exclusions).

TOP Factor scores journals on their adherence to ten transparency policies (http://cos.io/top). All polices are scored on a 0 to 3 scale, with the exception of policy 10 that has a maximum score of 2 . Journals are scored 0 on a policy if they only encourage adherence, or say nothing regarding the practice (original scoring rubric: https:// osf.io/t2yu5/).

1. Data citation - Journals are required to supply guides on how to cite data. Scores of 2 for stricter enforcement measures, and 3 for delaying article publication until citations standards are met.

2. Data transparency - Journal articles must state whether data is available and where. Scores of 2 for stricter enforcement measures such as requiring data is stored in an approved data repository, and 3 for delaying article publication until data is openly available and analysis results are independently reproduced.

3. Analytic code transparency - Journal articles must state whether code is available and where. Higher scores for stricter enforcement measures, following the standards of data transparency.

4. Materials transparency - Journal articles must state whether materials are available and where. Higher scores for stricter enforcement measures, following the standards of data transparency.

5. Design and analysis guidelines - Journal must clearly state design transparency standards. Scores for 2 for requiring adherence to transparency standards, and 3 for enforcing adherence, both during review and publication.

6. Study preregistration - Journal articles must state whether a preregistration exists and where it can be accessed. Scores of 2 if the journal also mandates access to preregistration during peer-review, and 3 for requiring preregistration (with associated link) and adding a pre-registration "badge" to articles.

7. Analysis plan preregistration - Same standards as study preregistration, but limited to the analysis phase.

8. Replication - Journal encourages replication studies. Scores of 2 for encouraging replication while implementing results blind review, and 3 for facilitating the submission of registered reports.

9. Registered reports - Journals state that an article's publication chance is not impacted by significance or novelty. Scores of 2 for implementing results blind reviews, and 3 for facilitating the submission of registered reports.

10. Open science badges - Journal awards articles with one or two open science badges. Higher score if all three badges are implemented. Maximum score of 2 . N.b. open science "badges" are labels journals apply to individuals articles (both in html and pdf versions) that signify that an article adheres to certain open science practices (Blohowiak et al., 2020; https://osf. io/tvyxz/).

We also obtained the TOP scores pertaining to the 346 journals so far assessed (top-factor.csv (v. 11) data obtained from https://osf.io/kgnva/, accessed on 202003-24). We compared our scores for herpetology journals to the overall presence of open and transparent practices in broader science. 
We used R v.3.5.3 (R Core Team, 2019) and R Studio v.1.2.1335 ( $R$ Studio Team, 2019), in conjunction with the dplyr v.0.8.4 (Wickham et al., 2019) and reshape2 v.1.4.3 (Wickham, 2007) packages for data manipulations, and ggplot2 v.3.2.1 (Wickham, 2016) for data visualisation. We made illustrative diagrams using Affinity Designer v.1.8.3.641 (Serif, 2020).

\section{RESULTS}

Nineteen journals listed on Scimago Journal and Country Rank had accessible author guideline URLs (see https:// osf.io/j4fyr/ [DOI: 10.17605/OSF.IO/J4FYR] for search and assessment results). Our assessment of these 19 journals revealed that herpetology journals fare poorly by TOP metrics. Sixteen of 19 journals assessed join $22 \%(75 / 346)$ of journals (so far assessed) failing to score a single TOP Factor point (Fig. 1). The overall mean (0.53 $\pm 0.37 ; n=19$; all \pm denote standard error) and median $(0 ; n=19)$ total scores for herpetology journals are lower than those derived from the TOP dataset (mean $=4.92$ \pm 0.28 ; median $=3 ; n=346$; Fig. 2 ). The highest scores achieved by herpetology journals were in the design and analysis guidelines.

\section{DISCUSSION}

While herpetology journals fare poorly regarding transparency policies, we have a distinct advantage in tackling practices that compromise replicability. We can quickly adopt methods, infrastructure, and guidelines from other fields; a wealth of options are available with transparency at their centre (Hampton et al., 2015; Parker et al., 2016), and we can prioritise the most successful and effort-effective solutions (Nuijten, 2019; Parker \& Nakagawa, 2014; Seigel, 2016; Voelkl et al., 2020). A concerted effort by the entire research community can maximise transparency, countering questionable research practices, while boosting the value of every study. Longer term changes will come with journal support, but authors can lead the charge irrespective of slow institution-level changes.

\section{Complete reporting}

Consistent and complete reporting of results is a simple way of improving transparency. Reporting practices often prove inadequate, missing key information (Archmiller et al., 2020; Cassey et al., 2004; Parker et al., 2016), but there are clear guides for what information to provide and how best to present that information (Fidler et al., 2018; Percie du Sert et al., 2019).

- Make a study discoverable (including full indexing by journals)

- Report a measure of spread with all means or medians

- Ensure all measures have units

- Include study location, use coordinates and state the coordinate system

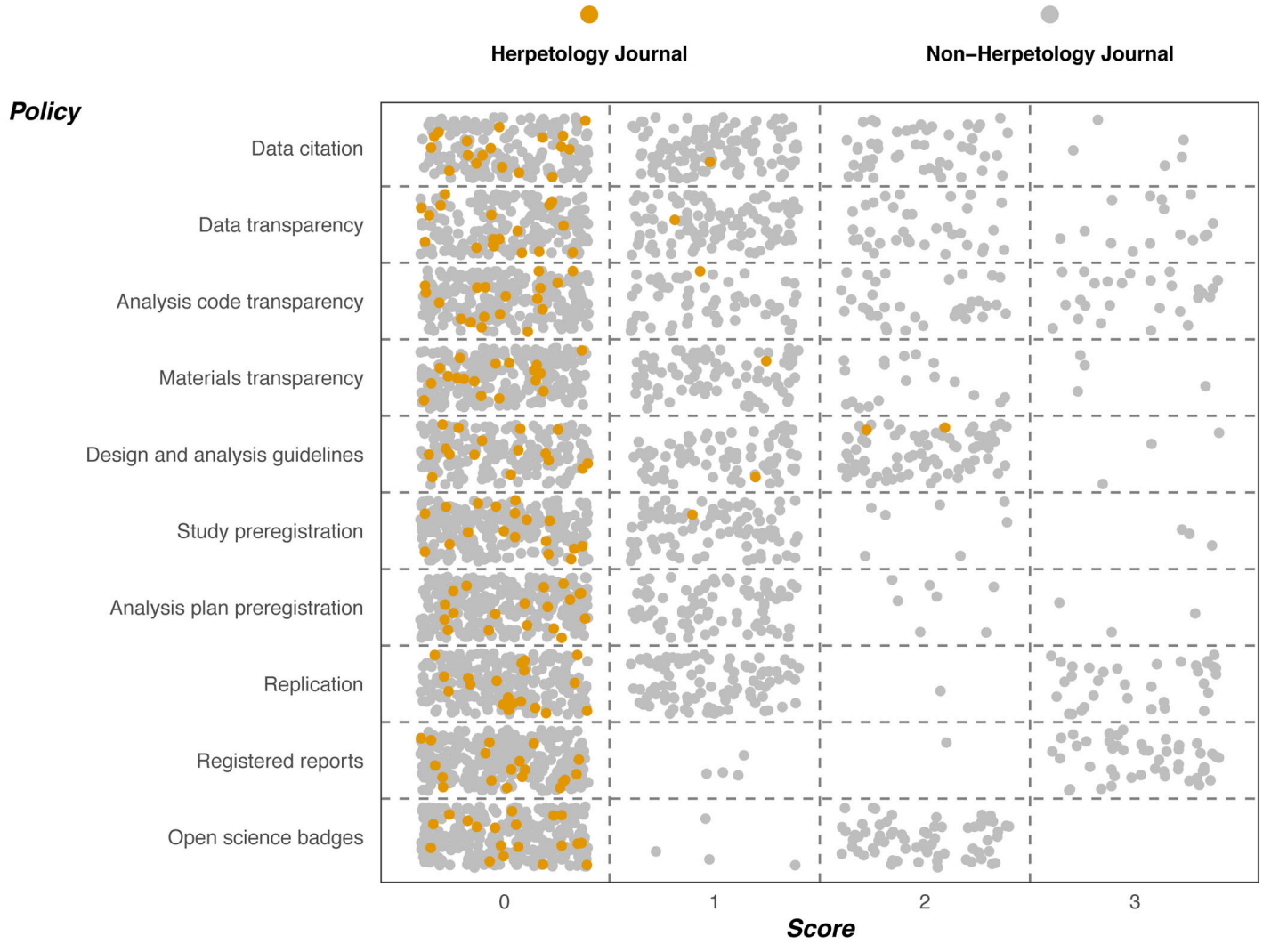

Figure 1. TOP scores of all 346 journals (grey points) plus 19 herpetology journals (orange points). Point locations are jittered within each score category; therefore, point position within the category is not indicative of decimal score. 


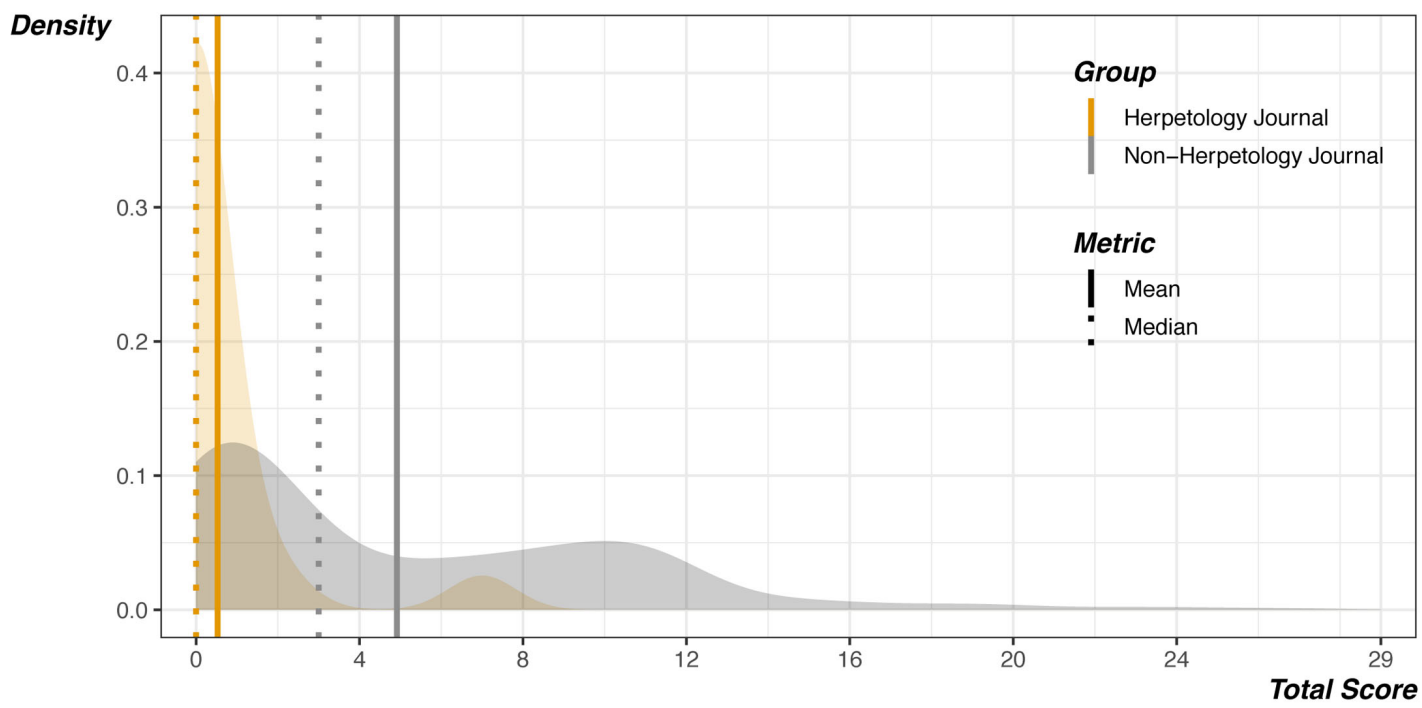

Figure 2. Density of total TOP scores of 19 herpetology journals (orange fill) and 346 non-herpetology journals (grey fill). Vertical lines indicate the mean (solid) and median (dotted) values for herpetology journals (orange) and nonherpetology journals (grey).

- Include spatial and temporal scale of study (i.e., study site and plot extents, study duration -be as specific as possible)

- Provide clear details on how means/medians are calculated from the total sample or subsets

- Report sample size and confidence/credible intervals for each statistical analysis

- Report all results regardless of outcome/significance

- Further suggestions can be found in Fidler et al. (2018) and Gerstner et al. (2017)

By following best practice for reporting we can maximise studies' utility and thus optimise for metaanalysis inclusion (Hillebrand \& Gurevitch, 2013; Nichols et al., 2019). Ensuring that methods and statistics are fully reported boosts reach and citations (Gerstner et al., 2017). Low sample sizes result in underpowered tests, variable effect sizes, and unreliable results that exacerbate false positives (Barto \& Rillig, 2012; Christie et al., 2019; Forstmeier et al., 2017; Jennions \& Møller, 2002). However, being able to combine these results from small studies will be even more valuable in cases where samples are limited by low-detection rates (Boback et al., 2020; Durso \& Seigel, 2015; Steen, 2010), technological limitations (Wolfe et al., 2018), and logistical obstacles (Christie et al., 2019). When fully and transparently reported, smaller studies expand and refine broader knowledge (Lemoine et al., 2016).

Full reporting through supplementary material can support meta-analyses. Many journals have restrictions on article length, often prompting us to prioritise the most pertinent findings and to deprioritise others. Making liberal use of supplementary material to report null results, visualise data distributions, and report fruitless exploratory analysis will keep null results present in the literature (Forstmeier et al., 2017). Without such results, meta-analyses can be biased towards positive significant findings (Jennions \& Møller, 2002), thus undermining the maturation of knowledge.

\section{Open data}

Data availability is the foundation of full reporting and expands study legacy (Gerstner et al., 2017). Extremely concerning findings from other disciplines connect the resistance to data sharing (even at review) to wider replication concerns; lack of data sharing hinders the detection of fabricated data (Czarnitzki et al., 2015; Miyakawa, 2020).

Sharing data on journal websites, or any website without long-term storage infrastructure, leaves data vulnerable to loss, change, and closure (e.g., Applied Herpetology ceased 2010, Hamadryad ceased 2012). The alternative is using dedicated data repositories (Whitlock, 2011). Data repositories (such as datadryad.org, Zenodo. org and OSF.io) are flexible regarding file types and size, as well as being considerably more durable, preserving data well after journals disappear (Whitlock, 2011).

The monetary cost of using these repositories is low to zero (Dryad: US\$120 but subject to waivers, Zenodo: Free, OSF: Free; Mislan et al., 2016). Researchers may be reticent to spend the time depositing data. But following existing guidance on data sharing (and ensuring its use by others) can substantially reduce the effort (Borer et al., 2009; Whitlock, 2011; Wilkinson et al., 2016). Using platforms like OSF during the data collection and collaborative phases can streamline final publication. In a few clicks researchers can switch repositories' visibility from private to public, immediately achieving two key points of open data:

- Store data on a stable repository

- Make data citable

Supplying adequate metadata (i.e., information describing the dataset) and storing data in nonproprietary formats (e.g., for datasheets use .csv versus .xlsx) maximises data utility. Metadata should fully explain all data columns, provide details on missing data values, and describe categorical variable codes, to fully enable 
third party data use. Using non-proprietary formats maintains data readability and boosts accessibility for researchers without expensive software. Improve data sheet readability by:

- Providing clear documentation

- Avoiding special characters (e.g., \%, \$, f, @)

- Restricting columns to a single data-type (e.g., binary, interval, categorical, continuous)

- Standardising date formats (ideally the international standard (ISO 8601) YYYY-MM-DD)

- Using descriptive file names

- Following further guidance by Borer et al. (2009).

Many data sharing best practices mirror those for data management, and easier to implement prior to data collection (Alston \& Rick, 2020; see Supplementary Table 1). As ecological datasets grow in size and complexity, metadata generation and data management are becoming increasingly necessary skills (Hernandez et al., 2012; Lewis et al., 2018). The British Ecological Society provides concise guidelines and checklists to promote a full suite of good data management practices (British Ecological Society, 2014).

\section{Open code}

Open data benefits are further bolstered by open reproducible analyses. The rise of programmable/ code-based analyses has enabled entirely recreatable workflows, from data curation to publication (Alston \& Rick, 2020); but providing open code is still lagging behind (Culina et al., 2020). A reproducible workflow is a phenomenal resource for reviewers and future researchers (Poisot, 2015), providing both transparency and guidance. Open data paired with code-based analyses enable reviewers to detect errors prior to publication, form the backbone of future studies, and facilitate replications (Mislan et al., 2016). When supplied alongside a paper, code offers a supplementary and more precise description of analysis that avoids the ambiguous language of prose methods sections (Archmiller et al., 2020; Ince et al., 2012). Open data and open code are both necessary for full computational reproducibility and the highest TOP scores for data and analytical transparency.

Version control is an added benefit of coding analysis: a way of recording all changes to files, with the ability to restore previous versions, and make changes simultaneously via branches (simultaneously existing versions of the same file). Online repositories further support version control such as GitHub (https://github. com/), Bitbucket (https://bitbucket.org/), and GitLab (https://www.gitlab.com/). These online platforms provide an additional back-up of files and joint work space for collaborators. Once analysis is completed, researchers can share analysis code via citable repositories with both reviewers and readers (Cooper et al., 2017; Poisot, 2015; White, 2015). However, GitHub and others are non-permanent, final code storage requires long-term solutions mirroring data storage options (Culina et al.,
2020). In its most reproducible form, code repositories can combine with Docker (https://www.docker.com/) or Binder (https://mybinder.org/) ensuring a re-runnable workspace (workspace being the environment and files associated with the code) capable of displaying analysis and results independent of the original researchers' workspace (Alston \& Rick, 2020).

Doubts over one's coding ability can make us reluctant to share. But realising that most code is cobbled together until it works counters this fear: "...if your code is good enough to do the job, then it is good enough to release." (Barnes, 2010). Errors in analysis are inevitable, even for organisations like the Met Office or NASA (Barnes, 2010; Ince et al., 2012). Sharing at the earliest opportunity offers the simplest solution to find and mitigate analytical errors. There is a wealth of options for learning and finding coding support (Carey \& Papin, 2018; Cooper et al., 2017; White, 2015; Wilson et al., 2014; Software Carpentry (http://software-carpentry. org/; see Supplementary Table 1).

\section{Resistance to sharing}

There is resistance to sharing, namely due to fears of scooping or competition (Anderson et al., 2007; Blumenthal et al., 2006), high cost-to-benefit, and exposing sensitive species. We feel these concerns can be easily mitigated within current frameworks.

Short-term data embargoes can counter scooping, where data is available to reviewers, and then opened once researchers have completed further desired analyses. Piwowar \& Vision (2013)'s findings suggest that one year embargoes would likely be adequate to guarantee the original researchers exclusive access, as third-party citations tend to occur at least two years after publishing. We still discourage most cases of embargoes. Immediate unfettered data access leads to immediate benefits. The costs to researchers are low, but the benefits are massive: boosting citation rates (Piwowar \& Vision, 2013), opening doors for new collaborations, and enabling large scale synthesis projects (Hampton et al., 2015; see Tucker et al. (2019) for an example of a study enabled by data storage). Sharing generates new questions, increases study reach, and informs future study/analysis design -resulting in better questions, quicker.

Protecting sensitive species' locations is a legitimate concern, as publication of new species localities has been implicated in the damaging exploitation of herpetofauna (Auliya et al., 2016; Stuart et al., 2006). Such concerns can be a legitimate reason for withholding geographic information. However, we encourage researchers to explicitly state limitations in data availability statements (as per TOP suggestions), while censoring the minimum amount of geographic information and leaving remainder accessible. As data accessibility becomes the default and statements on access mandatory (Aalbersberg et al., 2018), more authors will benefit from open data.

\section{Normative peer-review}

Peer review is a critical avenue for these practices to 


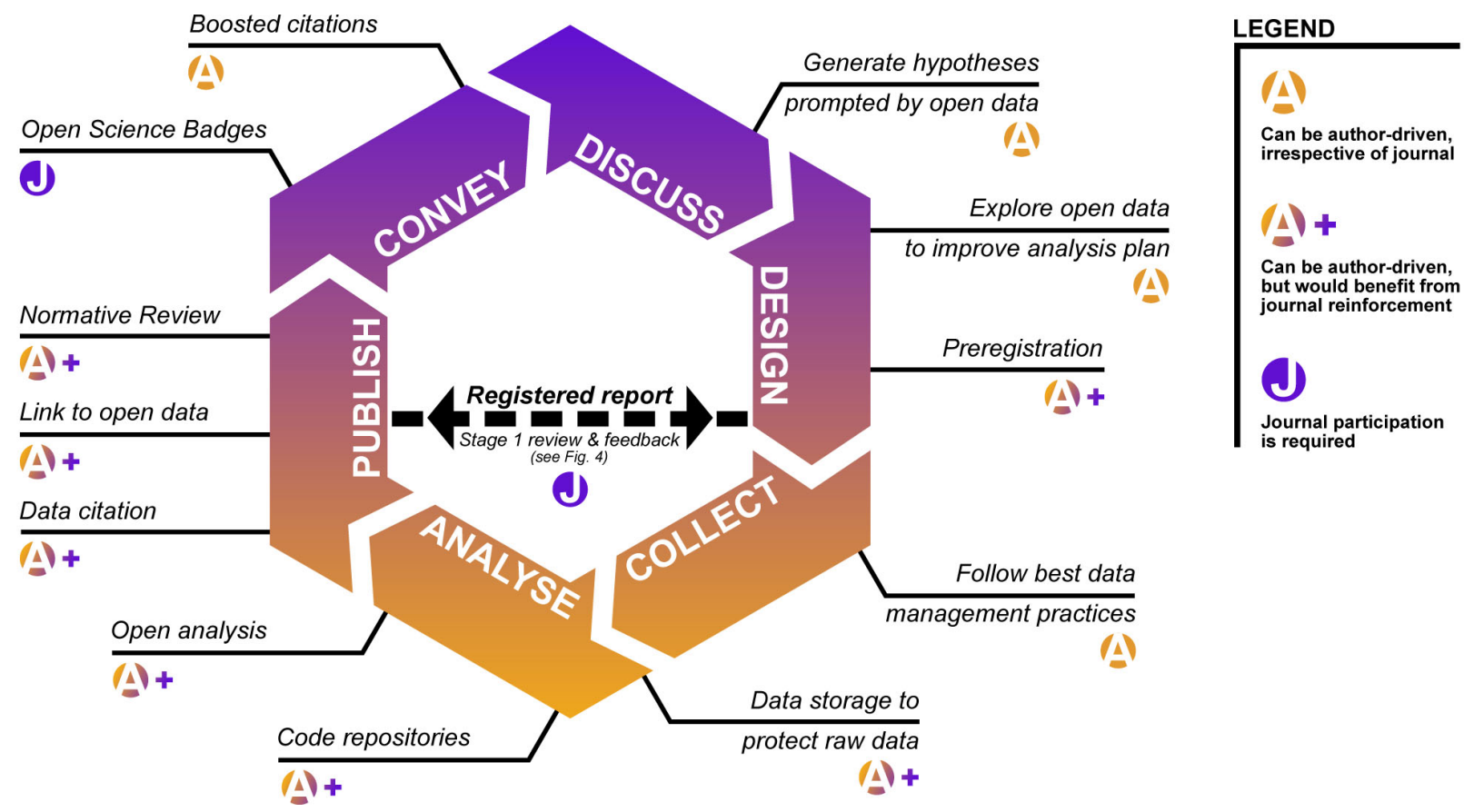

Figure 3. Diagram illustrating where practices and benefits of open science fit within the research cycle.

become more accepted and eventually standard (Morey et al., 2016; Poisot, 2015). We are provided with repeated opportunities to help each other refine manuscripts, and maximise studies' contributions. Referring back to guidelines for statistical reporting, data access, and reproducible analysis can ensure a consistent normative push towards a better body of literature (Morey et al., 2016). By leveraging checklists we improve the thoroughness of reviews and minimise the effort (Grey et al., 2020; Parker et al., 2018; Percie du Sert et al., 2019). The checklists compliment the improved reporting and transparency guidelines (paraphrased checklist from Parker et al., 2018):

- All sample sizes (and sample subsets) are fully reported

- Methods are sufficiently detailed for repeated analysis

- Statistical results are reported completely (e.g., all variables, tests, and transformations)

- Efforts taken to reduce unconscious biases

- Sample stopping rule stated (i.e., predefined sample size justification)

- Analysis designed prior to observing the data (e.g., a preregistration exists), otherwise described as exploratory

- Suitable research methods irrespective of results

- Sample size are capable of supporting authors' conclusions (e.g., tests are sufficiently powered)

- Estimated effect (and uncertainty) is considered in relation to the biological context

- Unexpected results are supported by strong evidence

Deficiencies in any of the above can highlight areas to improve reporting or where authors must acknowledge potential biases, rather than simply necessitating rejection
(Parker et al., 2018). By increasing transparency our ability to assess research quality is improved (Aalbersberg et al., 2018); open data and analysis enable us to suggest more targeted solutions, boosting the potency of the peer review process. Ideally journals can source specific code reviewers, who are fluent and familiar with the analysis code; code reviewers' impact would be bolstered if combined with a two-stage review system (see registered reports below).

\section{Reinforce with journal policy}

Journals can promote transparent policies by modifying their author guidelines. Journal-level enforcement (Roche et al., 2015), and clear guidelines for editors and authors (Christian et al., 2020), are required because data access requests are rarely fulfilled without enforcement (Archmiller et al., 2020). Similar enforcement will likely improve transparency in data citation and analysis code (Culina et al., 2020).

Other journals have demonstrated how policy changes can rapidly modify publishing practice (Nosek et al., 2012). Several herpetology journals already require mandatory data deposition for genetic data (e.g., African Journal of Herpetology, Copeia [renamed to Ichthyology and Herpetology], Herpetology Notes, Salamandra, and South American Journal of Herpetology). Therefore, improving TOP scores only requires expanding existing policy rather than introducing new rules. Once journal policy has expanded to target pro-transparency practices, authors and peer reviewers can be supplied with checklists to guide best practices (e.g., those provided by PeerJ, Herpetological Conservation and Biology, Nature Communications).

The discipline of psychology has led the charge toward open science practices, showing successful implementations drove normative change. For instance, 
the journal Psychological Science is one of a number of journals that began using "open science badges" to label papers that implemented practices such as data archiving. These badges not only help readers identify transparent and reliable papers, but they may incentivise authors to pursue open science to earn the badges (Blohowiak et al., 2020; Kidwell et al., 2016).

\section{Publish negative results and replications}

All herpetology journals scored zero in the replication TOP policy (Fig. 1). Following the recommendations of TOP, journals must be open to publishing replications and negative results (Nakagawa \& Parker, 2015), dispensing with statements encouraging novelty. If journals can relieve the pressure for positive/significant results, the incentives to undertake questionable practices will cease to exist (Nilsen et al., 2020), and counter publication biases (Nichols et al., 2019). Replications do not suffer the lower citations rates that journals fear (Forstmeier et al., 2017), and researchers welcome them (Fraser et al., 2019). We argue that replication studies in herpetology would accumulate citations faster (along with original study) as they further validate or refute findings.

Registered reports are the most direct way to counter publication bias (Allen \& Mehler, 2018). Registered reports remove the results from the assessment of publication-worthiness, via a two-stage peer review: stage 1 assesses the study methodology prior to interacting with the data, stage 2 assesses whether the researchers followed their proposed methodology (Fig. 4). Critically, the journal decides whether to accept the publication at stage 1, via an "in principle acceptance", meaning any decision is only contingent on a solid study design, not novel findings or significant results. The stage 1 peer review has vast benefits for researchers: we can correct weaker methods and analyses, we can identify journal guideline conflicts, and we can improve methods based on overlooked literature, prior to expending time and money on experimentation (Dirnagl, 2020).

Disassociating the results from publishing decisions is especially valuable for countering questionable research practices ( $p$-hacking, HARKing, and cherrypicking). As the methods are decided prior to seeing the data, researchers' analytical flexibility becomes limited and the distinction between hypothesis testing versus exploratory results becomes clearer. In addition, obtaining a peer review prior to data collection can generate new hypotheses, approaches for analysing the data, and new useful covariates.

While registered reports require journal collaboration, authors can begin restructuring their research workflow with preregistrations. Preregistrations lack registered reports' level of rigor (namely the stage 1 peer review), and the protection against journals' prioritisation of positive/significant results. But they do present a powerful framework considering study design flexibility. Preregistrations produce a record of time-stamped a priori hypotheses that aids later peer-reviewers to identify exploratory analysis and selective reporting (Parker et al., 2019; Toth et al., 2020). Herpetology journal adoption of registered reports is currently nonexistent, so authors can leverage preregistration to improve their own practice.

The lack of incentives to review can create an environment where submitted publications outstrip available reviewers (Fox \& Petchey, 2010; Peres-Neto, 2016); therefore, an additional review stage may seem like a further burden. Using a wider more diverse pool of career researchers would lessen the pressure on individual peer reviewers (e.g., the Early Career Reviewer Database created by Susan Perkins, Curator \& Professor, AMNH: https://sites.google.com/view/ecrdatabase/ home; Garisto, 2020; Seigel, 2016), and multi-stage review will catch issues earlier, thus reducing the overall effort of review during publishing (Parker et al., 2019).

\section{Closing remarks}

We highlight three key avenues to enhance replicability in herpetology: personal practice, open science, and journal policy. The first requires author awareness. Only by recognising the incentives that promote questionable practices can we actively shun them. Second, share widely and freely, promote openness and reward transparent reporting. If we make transparency core to publishing and peer review, we can steer practices towards a system that amplifies error detection, provides more

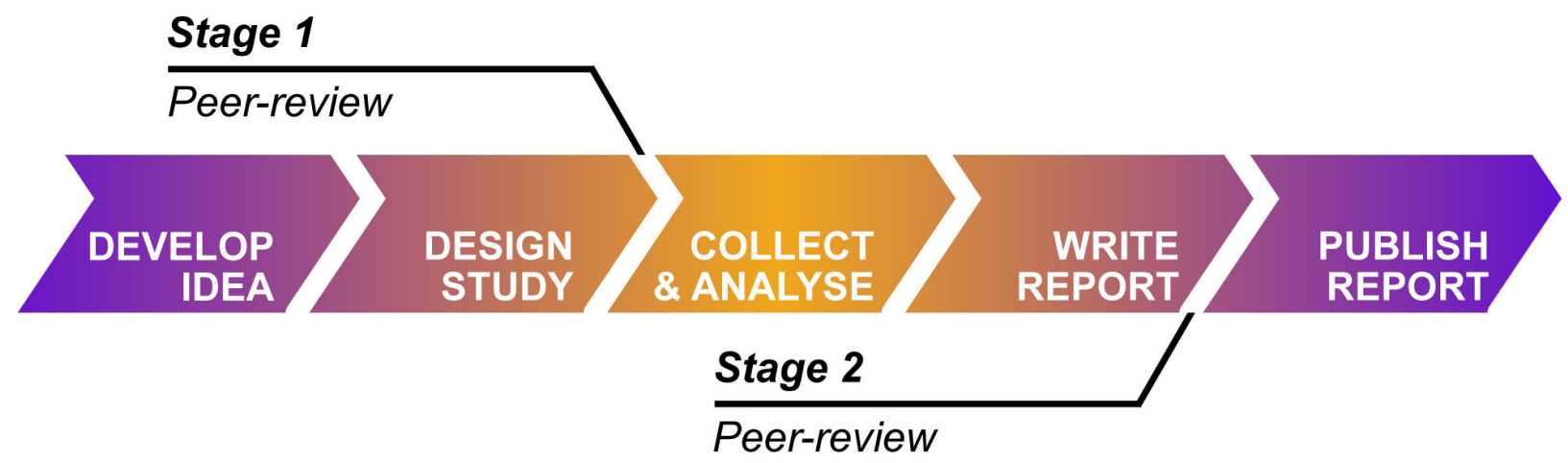

Figure 4. Diagram illustrating the registered report timeline from idea to final publication. Redrawn from OSF summary on registered reports: https://osf.io/rr/. 
insightful refinements, and builds stronger foundations for future studies. Finally, support journals promoting transparency, but do not allow enforcement deficiencies to prevent us from following our own best practice to maximise transparency. The publishing system may be slow to change -we, as authors, editors and reviewers, must lead. We can all enhance herpetology by adopting and benefiting from open science.

\section{Data and code availability}

Supplementary Table 1 contains open science resources (Supplementary Table 1 - Open science resources. csv), the Scimago Journal and Country Rank search results (SJR - Journal Search Results 2020-02-21.pdf), assessment results of herpetology journals (top-herpassessment_2020-03-16.csv \& top-herp-assessment metadata 2020-03-16.csv), the overall TOP dataset used (top-factor_2020-03-24.csv \& top-factor metadata_2020-03-24.csv), $R$ code to summarise the data and produce figures (Summary of TOP scores.R), and diagrams of the research cycle and registered reports are available at https://osf.io/j4fyr/, DOI: 10.17605/OSF.IO/ J4FYR.

\section{Contributions}

Conceptualisation - B.M.M. and C.T.S., Formal analyses - B.M.M., Investigation - B.M.M., Writing - Original Draft - B.M.M., Writing - Review \& Editing - B.M.M. and C.T.S., Visualisation - B.M.M.

\section{ACKNOWLEDGEMENTS}

We thank the Suranaree University of Technology (SUT) School of Biology, Institute of Science for financial and logistical support. We thank the SUT Institute of Research and Development for financial support. We thank Matthew Crane and Inês Silva for countless discussions on data and analytical practice. We thank Dan Quintana and James Heathers for their Everything Hertz podcast which drew our attention to the reproducibility crises in a number of scientific fields promoting us to consider the importance of open science. We thank Aubrey Alamshah for reviewing the manuscript. We thank Dr. Julia Riley and Dr. Tim Parker for their extremely thorough reviews that greatly improved the manuscript.

\section{REFERENCES}

Aalbersberg, Ij. J., Appleyard, T., Brookhart, S., Carpenter, T., Clarke, M., Curry, S., Dahl, J., DeHaven, A.C., Eich, E., Franko, M., ... Vazire, S. (2018). Making Science Transparent By Default: Introducing the TOP Statement. OSF Preprints. DOI: 10.31219/osf.io/sm78t

Allen, C.P.G. \& Mehler, D.M.A. (2018). Open Science challenges, benefits and tips in early career and beyond. PsyArXiv. DOI: 10.31234/osf.io/3czyt

Alston, J. \& Rick, J. (2020). A Beginner's Guide to Conducting Reproducible Research. EcoEvoRxiv. DOI: 10.32942/osf.io/ h5r6n

Anderson, M.S., Ronning, E.A., De Vries, R. \& Martinson,
B.C. (2007). The Perverse Effects of Competition on Scientists' Work and Relationships. Science and Engineering Ethics 13(4), 437-461. DOI: 10.1007/s11948-007-9042-5 Anvari, F. \& Lakens, D. (2018). The replicability crisis and public trust in psychological science. Comprehensive Results in Social Psychology 3(3), 266-286. DOI: 10.1080/23743603.2019.1684822

Archmiller, A.A., Johnson, A.D., Nolan, J., Edwards, M., Elliott, L.H., Ferguson, J.M., lannarilli, F., Vélez, J., Vitense, K., Johnson, D.H. \& Fieberg, J. (2020). Computational Reproducibility in The Wildlife Society's Flagship Journals. The Journal of Wildlife Management, jwmg.21855. DOI: 10.1002/jwmg.21855

Auliya, M., Altherr, S., Ariano-Sanchez, D., Baard, E.H., Brown, C., Brown, R.M., Cantu, J.C., Gentile, G., Gildenhuys, P., Henningheim, E.,... Ziegler, T. (2016). Trade in live reptiles, its impact on wild populations, and the role of the European market. Biological Conservation 204, 103-119. DOI: 10.1016/j.biocon.2016.05.017

Barnes, N. (2010). Publish your computer code: It is good enough. Nature, 467(7317), 753-753. DOI: $10.1038 / 467753 a$

Barto, E.K. \& Rillig, M.C. (2012). Dissemination biases in ecology: Effect sizes matter more than quality. Oikos 121(2), 228-235. DOI: 10.1111/j.1600-0706.2011.19401.x

Blohowiak, B. B., Cohoon, J., de-Wit, L., Eich, E., Farach, F.J., Hasselman, F., Holcombe, A.O., Humphreys, M., Lewis, M., Nosek, B.A. et al. (2020). Badges to Acknowledge Open Practices. OSF. osf.io/tvyxz

Blumenthal, D., Campbell, E. G., Gokhale, M., Yucel, R., Clarridge, B., Hilgartner, S. \& Holtzman, N.A. (2006). Data withholding in genetics and the other life sciences: Prevalences and predictors. Academic Medicine: Journal of the Association of American Medical Colleges 81(2), 137-145. DOI: 10.1097/00001888-200602000-00008

Boback, S.M., Nafus, M.G., Yackel Adams, A.A. \& Reed, R.N. (2020). Use of visual surveys and radiotelemetry reveals sources of detection bias for a cryptic snake at low densities. Ecosphere, 11(1). DOI: 10.1002/ecs2.3000

Borer, E. T., Seabloom, E.W., Jones, M.B. \& Schildhauer, M. (2009). Some Simple Guidelines for Effective Data Management. Bulletin of the Ecological Society of America 90(2), 205-214. DOI: 10.1890/0012-9623-90.2.205

Brembs, B. (2018). Prestigious Science Journals Struggle to Reach Even Average Reliability. Frontiers in Human Neuroscience 12(February), 1-7. https://doi.org/10.3389/ fnhum.2018.00037

Brembs, B. (2019). Reliable novelty: New should not trump true. PLOS Biology 17(2), e3000117. DOI: 10.1371/journal. pbio.3000117

British Ecological Society. (2014). A Guide to Data Management in Ecology and Evolution (BES Guides to Better Science). British Ecological Society.

Cairo, A.H., Green, J.D., Forsyth, D.R., Behler, A.M.C., \& Raldiris, T.L. (2020). Gray (Literature) Matters: Evidence of Selective Hypothesis Reporting in Social Psychological Research. Personality and Social Psychology Bulletin, 014616722090389. DOI: 10.1177/0146167220903896

Carey, M.A. \& Papin, J.A. (2018). Ten simple rules for biologists learning to program. PLOS Computational Biology 14(1), e1005871. DOI: 10.1371/journal.pcbi.1005871 
Cassey, P., Ewen, J.G., Blackburn, T.M. \& Møller, A.P. (2004). A survey of publication bias within evolutionary ecology. Proceedings of the Royal Society of London. Series B: Biological Sciences, 271(suppl_6). DOI: 10.1098/ rsbl.2004.0218

Christian, T.-M., Gooch, A., Vision, T. \& Hull, E. (2020). Journal data policies: Exploring how the understanding of editors and authors corresponds to the policies themselves. PLOS ONE 15(3), e0230281. DOI: 10.1371/journal.pone.0230281

Christie, A.P., Amano, T., Martin, P.A., Shackelford, G.E., Simmons, B.I. \& Sutherland, W.J. (2019). Simple study designs in ecology produce inaccurate estimates of biodiversity responses. Journal of Applied Ecology 56(12), 2742-2754. DOI: 10.1111/1365-2664.13499

Clark, T.D., Raby, G.D., Roche, D.G., Binning, S.A., Speers-Roesch, B., Jutfelt, F. \& Sundin, J. (2020). Ocean acidification does not impair the behaviour of coral reef fishes. Nature 577(7790), 370-375. DOI: 10.1038/s41586-019-1903-y

Cooper, N., Hsing, P.-Y., Croucher, M., Graham, L., James, T., Krystalli, A. \& Michonneau, F. (2017). A Guide to Reproducible Code in Ecology and Evolution (British Ecological Society) [BES Guides to Better Science].

Culina, A., van den Berg, I., Evans, S. \& Sánchez-Tójar, A. (2020). Low availability of code in ecology: A call for urgent action. PLOS Biology 18(7), e3000763. DOI: 10.1371/journal. pbio.3000763

Czarnitzki, D., Grimpe, C. \& Pellens, M. (2015). Access to research inputs: Open science versus the entrepreneurial university. The Journal of Technology Transfer 40(6), 10501063. DOI: 10.1007/s10961-015-9392-0

Dirnagl, U. (2020). Preregistration of exploratory research: Learning from the golden age of discovery. PLOS Biology 18(3), e3000690. DOI: 10.1371/journal.pbio.3000690

Durso, A.M. \& Seigel, R.A. (2015). A Snake in the Hand is Worth 10,000 in the Bush. Journal of Herpetology 49(4), 503-506. DOI: 10.1670/15-49-04.1

Fanelli, D. (2010). Do Pressures to Publish Increase Scientists' Bias? An Empirical Support from US States Data. PLOS ONE 5(4), e10271. DOI: 10.1371/journal.pone.0010271

Fanelli, D. (2012). Negative results are disappearing from most disciplines and countries. Scientometrics 90(3), 891-904. DOI: 10.1007/s11192-011-0494-7

Fidler, F., Fraser, H., Mccarthy, M.A. \& Game, E.T. (2018). Improving the transparency of statistical reporting in Conservation Letters. Conservation Letters 11(e12453), 1-4. DOI: $10.1111 /$ conl.12453

Forstmeier, W., Wagenmakers, E.-J. \& Parker, T.H. (2017). Detecting and avoiding likely false-positive findings - a practical guide: Avoiding false-positive findings. Biological Reviews 92(4), 1941-1968. DOI: 10.1111/brv.12315

Fox, J. \& Petchey, O.L. (2010). Pubcreds: Fixing the Peer Review Process by "Privatizing" the Reviewer Commons. Bulletin of the Ecological Society of America 91(3), 325-333. DOI: 10.1890/0012-9623-91.3.325

Fraser, H., Parker, T., Fidler, F. \& Barnett, A. (2019). The role of replication studies in ecology. EcoEvoRxiv. DOI: 10.32942/ osf.io/4cuwp

Fraser, H., Parker, T., Nakagawa, S., Barnett, A. \& Fidler, F. (2018). Questionable research practices in ecology and evolution. PLOS ONE 13(7), e0200303. DOI: 10.1371/journal. pone. 0200303
Freedman, L.P., Cockburn, I.M. \& Simcoe, T.S. (2015). The Economics of Reproducibility in Preclinical Research. PLOS Biology 13(6), e1002165. DOI: 10.1371/journal. pbio. 1002165

Garisto, D. (2020). Diversifying peer review by adding junior scientists. Nature Index. Gerstner, K., Moreno-Mateos, D., Gurevitch, J., Beckmann, M., Kambach, S., Jones, H. P. \& Seppelt,

R. (2017). Will your paper be used in a meta-analysis? Make the reach of your research broader and longer lasting. Methods in Ecology and Evolution 8(6), 777-784. DOI: 10.1111/2041-210X.12758

Grainger, M., Bolam, F. C., stewart, G. \& Nilsen, E. B. (2019). Evidence synthesis for tackling research waste. EcoEvoRxiv. DOI: 10.32942/osf.io/42fkh

Grey, A., Bolland, M. J., Avenell, A., Klein, A. A. \& Gunsalus, C. K. (2020). Check for publication integrity before misconduct. Nature 577(7789), 167-169. DOI: 10.1038/d41586-01903959-6

Hampton, S.E., Anderson, S.S., Bagby, S.C., Gries, C., Han, X., Hart, E.M., Jones, M.B., Lenhardt, W.C., MacDonald, A., Michener, W.K., Mudge, J., Pourmokhtarian, A., Schildhauer, M.P., Woo, K.H. \& Zimmerman, N. (2015). The Tao of open science for ecology. Ecosphere 6(7), art120. DOI: 10.1890/ ES14-00402.1

Hernandez, R.R., Mayernik, M.S., Murphy-Mariscal, M.L. \& Allen, M.F. (2012). Advanced Technologies and Data Management Practices in Environmental Science: Lessons from Academia. BioScience 62(12), 1067-1076. DOI: 10.1525/bio.2012.62.12.8

Hillebrand, H. \& Gurevitch, J. (2013). Reporting standards in experimental studies. Ecology Letters 16(12), 1419-1420. DOI: 10.1111/ele.12190

Ince, D.C., Hatton, L. \& Graham-Cumming, J. (2012). The case for open computer programs. Nature 482(7386), 485-488. DOI: 10.1038/nature10836

Jennions, M.D. \& Møller, A.P. (2002). Publication bias in ecology and evolution: An empirical assessment using the trim and fill' method. Biological Reviews of the Cambridge Philosophical Society 77(2), 211-222. DOI: 10.1017/ S1464793101005875

Kaplan, R.M. \& Irvin, V.L. (2015). Likelihood of Null Effects of Large NHLBI Clinical Trials Has Increased over Time. PLOS ONE 10(8), e0132382. DOI: 10.1371/journal.pone.0132382

Kelly, C.D. (2019). Rate and success of study replication in ecology and evolution. PeerJ 7, e7654. DOI: 10.7717/ peerj.7654

Kidwell, M.C., Lazarević, L.B., Baranski, E., Hardwicke, T. E., Piechowski, S., Falkenberg, L.-S., Kennett, C., Slowik, A., Sonnleitner, C., Hess-Holden, C., Errington, T.M., Fiedler, S. \& Nosek, B.A. (2016). Badges to Acknowledge Open Practices: A Simple, Low-Cost, Effective Method for Increasing Transparency. PLoS Biology 14(5). DOI: 10.1371/ journal.pbio.1002456

Lemoine, N.P., Hoffman, A., Felton, A.J., Baur, L., Chaves, F., Gray, J., Yu, Q. \& Smith, M.D. (2016). Underappreciated problems of low replication in ecological field studies. Ecology 97(10), 2554-2561. DOI: 10.1002/ecy.1506

Lewis, K.P., Vander Wal, E. \& Fifield, D.A. (2018). Wildlife biology, big data, and reproducible research: Reproducible Research. Wildlife Society Bulletin 42(1), 172-179. DOI: 


\subsection{2/wsb.847}

Markowetz, F. (2015). Five selfish reasons to work reproducibly. Genome Biology 16(1), 274. DOI: 10.1186/s13059-015 0850-7

Mislan, K.A.S., Heer, J.M. \& White, E.P. (2016). Elevating The Status of Code in Ecology. Trends in Ecology \& Evolution 31(1), 4-7. DOI: 10.1016/j.tree.2015.11.006

Miyakawa, T. (2020). No raw data, no science: Another possible source of the reproducibility crisis. Molecular Brain 13(1), 24, s13041-020-0552-2. DOI: 10.1186/s13041-020-0552-2

Morey, R.D., Chambers, C.D., Etchells, P. J., Harris, C.R., Hoekstra, R., Lakens, D., Lewandowsky, S., Morey, C.C., Newman, D. P., Schönbrodt,.. Zwaan, R.A. (2016). The Peer Reviewers' Openness Initiative: Incentivizing open research practices through peer review. Royal Society Open Science 3(1), 150547. DOI: 10.1098/rsos.150547

Nakagawa, S. \& Parker, T.H. (2015). Replicating research in ecology and evolution: Feasibility, incentives, and the costbenefit conundrum. BMC Biology 13(1), 88. DOI: 10.1186/ s12915-015-0196-3

Nichols, J.D., Kendall, W.L. \& Boomer, G.S. (2019). Accumulating evidence in ecology: Once is not enough. Ecology and Evolution 9(24), 13991-14004. DOI: 10.1002/ece3.5836

Nilsen, E.B., Bowler, D.E. \& Linnell, J.D.C. (2020). Exploratory and confirmatory research in the open science era. Journal of Applied Ecology, 1365-2664.13571. DOI: 10.1111/13652664.13571

Nosek, B.A., Alter, G., Banks, G.C., Borsboom, D., Bowman, S.D., Breckler, S.J., Buck, S., Chambers, C.D., Chin, G., Christensen, G.,... Yarkoni, T. (2015). Promoting an open research culture. Science 348(6242), 1422-1425. DOI: 10.1126/science. aab2374

Nosek, B., Spies, J.R. \& Motyl, M. (2012). Scientific Utopia: II. Restructuring Incentives and Practices to Promote Truth Over Publishability [Data set]. In Perspectives on Psychological Science 7(6), 615-631. DOI: 10.1177/1745691612459058

O’Boyle, E.H., Banks, G.C. \& Gonzalez-Mulé, E. (2014). The Chrysalis Effect: How Ugly Initial Results Metamorphosize Into Beautiful Articles. Journal of Management 43(2), 376399. DOI: $10.1177 / 0149206314527133$

Open Science Collaboration. (2015). Estimating the reproducbility of psychological science. Science 349(6251), aac4716aac4716. DOI: 10.1126/science.aac4716

Parker, T., Fraser, H. \& Nakagawa, S. (2019). Making conservation science more reliable with preregistration and registered reports. Conservation Biology 33(4), 747-750. DOI: 10.1111/cobi.13342

Parker, T.H., Forstmeier, W., Koricheva, J., Fidler, F., Hadfield, J. D., Chee, Y. ., Kelly, C.D., Gurevitch, J. \& Nakagawa, S. (2016). Transparency in Ecology and Evolution: Real Problems, Real Solutions. Trends in Ecology \& Evolution 31(9), 711-719. DOI: 10.1016/j.tree.2016.07.002

Parker, T.H., Griffith, S.C., Bronstein, J.L., Fidler, F., Foster, S., Fraser, H., Forstmeier, W., Gurevitch, J., Koricheva, J., Seppelt, R., Tingley, M.W. \& Nakagawa, S. (2018). Empowering peer reviewers with a checklist to improve transparency. Nature Ecology \& Evolution 2(6), 929-935. DOI: 10.1038/s41559018-0545-z

Percie du Sert, N., Hurst, V., Ahluwalia, A., Alam, S., Avey, M. T., Baker, M., Browne, W. J., Clark, A., Cuthill, I. C., Dirnagl,... Würbel, H. (2019). The ARRIVE guidelines 2019: Updated guidelines for reporting animal research. BioRxiv. DOI: 10.1101/703181

Peres-Neto, P. R. (2016). Will technology trample peer review in ecology? Ongoing issues and potential solutions. Oikos 125(1), 3-9. DOI: 10.1111/oik.02956

Piwowar, H.A. \& Vision, T.J. (2013). Data reuse and the open data citation advantage. PeerJ 1, e175. DOI: 10.7717/ peerj.175

Poisot, T. (2015). Best publishing practices to improve user confidence in scientific software. Ideas in Ecology and Evolution 8. DOI: 10.4033/iee.2015.8.8.f

R Core Team. (2019). R: A language and environment for statistical computing. R Foundation for Statistical Computing. https://www.r-project.org/

R Studio Team. (2019). RStudio: Integrated Development Environment for R. RStudio, Inc. http://www.rstudio.com/

Rice, D.B., Raffoul, H., Ioannidis, J.P.A. \& Moher, D. (2020). Academic criteria for promotion and tenure in biomedical sciences faculties: Cross sectional analysis of international sample of universities. BMJ, m2081. DOI: 10.1136/bmj. m2081

Roche, D.G., Amcoff, M., Morgan, R., Sundin, J., Finnøen, M. H., Lawrence, M. J., Henderson, E., Speers-Roesch, B., Brown, C., Clark, T. D., Bshary, R., Jutfelt, F. \& Binning, S.A. (2020). Behavioural lateralisation in a detour test is not repeatable in fishes. EcoEvoRxiv, 62. DOI: 10.32942/osf. io/6 kcwa

Roche, D.G., Kruuk, L.E.B., Lanfear, R. \& Binning, S.A. (2015). Public Data Archiving in Ecology and Evolution: How Well Are We Doing? PLOS Biology 13(11), e1002295. DOI: /10.1371/journal.pbio.1002295

Sánchez-Tójar, A., Nakagawa, S., Sánchez-Fortún, M., Martin, D. A., Ramani, S., Girndt, A., Bókony, V., Kempenaers, B., Liker, A., Westneat, D. F., Burke, T. \& Schroeder, J. (2018). Metaanalysis challenges a textbook example of status signalling and demonstrates publication bias. ELife 7, e37385. DOI: 10.7554/eLife. 37385

Scheel, A.M., Schijen, M. \& Lakens, D. (2020). An excess of positive results: Comparing the standard Psychology literature with Registered Reports. PsyArXiv. DOI: 10.31234/ osf.io/p6e9c

Schnitzer, S.A. \& Carson, W. P. (2016). Would Ecology Fail the Repeatability Test? BioScience 66(2), 98-99. DOI: 10.1093/ biosci/biv176

Seigel, R.A. (2016). The Future of Publishing Herpetological Research: Peer Review, "Pre-Publications", and Openness and Transparency of Data. Journal of Herpetology 50(4), 497-501. DOI: 10.1670/16-120

Serif. (2020). Affinity Designer (1.8.3.641) [Windows]. Serif Europe Ltd.

Steen, D. A. (2010). Snakes in the grass: Secretive natural histories defy both conventional and progressive statistics. Herpetological Conservation and Biology 5(2), 183-188.

Stuart, B.L., Rhodin, A.G.J., Grismer, L.L. \& Hansel, T. (2006). Scientific description can imperil species. Science, 312(5777), 1137. DOI: 10.1126/science.312.5777.1137b

Toth, A.A., Banks, G.C., Mellor, D., O'Boyle, E.H., Dickson, A., Davis, D.J., DeHaven, A., Bochantin, J. \& Borns, J. (2020). Study Preregistration: An Evaluation of a Method for Transparent Reporting. Journal of Business and Psychology. DOI: 10.1007/s10869-020-09695-3 
Tucker, M.A., Alexandrou, O., Bierregaard, R.O., Bildstein, K. L., Böhning-Gaese, K., Bracis, C., Brzorad, J.N., Buechley, E. R., Cabot, D., Calabrese,... Mueller, T. (2019). Large birds travel farther in homogeneous environments. Global Ecology and Biogeography 28(5), 576-587. DOI: 10.1111/ geb.12875

Wang, D., Forstmeier, W., Ihle, M., Khadraoui, M., Jerónimo, S., Martin, K. \& Kempenaers, B. (2018). Irreproducible textbook "knowledge": The effects of color bands on zebra finch fitness: COLOR BANDS HAVE NO EFFECT ON FITNESS IN ZEBRA FINCHES. Evolution 72(4), 961-976. DOI: 10.1111/ evo.13459

Ware, J.J. \& Munafò, M.R. (2015). Significance chasing in research practice: Causes, consequences and possible solutions: Significance chasing. Addiction 110(1), 4-8. DOI: 10.1111/add.12673

White, E. (2015). Some thoughts on best publishing practices for scientific software. Ideas in Ecology and Evolution 8. DOI: 10.4033/iee.2015.8.9.c

Whitlock, M. C. (2011). Data archiving in ecology and evolution: Best practices. Trends in Ecology \& Evolution 26(2), 61-65. DOI: 10.1016/j.tree.2010.11.006
Wickham, H. (2007). Reshaping Data with the \{reshape\} Package. Journal of Statistical Software 21(12), 1-20.

Wickham, H. (2016). ggplot2: Elegant graphics for data analysis. Springer-Verlag New York.

Wickham, H., François, R., Henry, L. \& Müller, K. (2019). dplyr: A Grammar of Data Manipulation (0.8.3) [Computer software].

Wilkinson, M. D., Dumontier, M., Aalbersberg, Ij. J., Appleton, G., Axton, M., Baak, A., Blomberg, N., Boiten, J.-W., da Silva Santos, L. B.,... Mons, B. (2016). The FAIR Guiding Principles for scientific data management and stewardship. Scientific Data 3(1), 160018. DOI: 10.1038/sdata.2016.18

Wilson, G., Aruliah, D.A., Brown, C.T., Chue Hong, N.P., Davis, M., Guy, R.T., Haddock, S. H.D., Huff, K.D., Mitchell, I.M., Plumbley, M.D., Waugh, B., White, E. P. \& Wilson, P. (2014). Best Practices for Scientific Computing. PLoS Biology, 12(1), e1001745. DOI: 10.1371/journal.pbio.1001745

Wolfe, A.K., Fleming, P.A. \& Bateman, P.W. (2018). Impacts of translocation on a large urban-adapted venomous snake. Wildlife Research. DOI: 10.1071/WR17166

Accepted: 14 October 2020 